Research Article

\title{
Investigation of the Mechanism of Traditional Chinese Medicines in Angiogenesis through Network Pharmacology and Data Mining
}

\author{
Wingyan Yun $\mathbb{D},{ }^{1,2}$ Wenchao Dan $\mathbb{D},{ }^{1,2}$ Jinlei Liu $\mathbb{D},{ }^{1}$ Xinyuan Guo $\mathbb{D}^{3},{ }^{3}$ Min Li $\mathbb{D}$, ${ }^{1}$ \\ and Qingyong $\mathrm{He} \mathbb{C}^{1}$ \\ ${ }^{1}$ Guang'anmen Hospital, China Academy of Chinese Medical Sciences, Beijing 100053, China \\ ${ }^{2}$ Graduate School of Beijing University of Chinese Medicine, Beijing 100029, China \\ ${ }^{3}$ Cancer Hospital Chinese Academy of Medical Sciences, Beijing 100021, China
}

Correspondence should be addressed to Qingyong He; heqingyongg@163.com

Received 12 February 2021; Revised 20 March 2021; Accepted 15 April 2021; Published 29 April 2021

Academic Editor: Siba shanak

Copyright (C) 2021 Wingyan Yun et al. This is an open access article distributed under the Creative Commons Attribution License, which permits unrestricted use, distribution, and reproduction in any medium, provided the original work is properly cited.

Although traditional Chinese medicine is effective and safe for the treatment of angiogenesis, the in vivo intervention mechanism is diverse, complex, and largely unknown. Therefore, we aimed to explore the active ingredients of traditional Chinese medicine and their mechanisms of action against angiogenesis. Data on angiogenesis-related targets were collected from GeneCards, Therapeutic Target Database, Online Mendelian Inheritance in Man, DrugBank, and DisGeNET. These were matched to related molecular compounds and ingredients in the traditional Chinese medicine system pharmacology platform. The data were integrated and based on the condition of degree $>1$, and relevant literature, target-compound, compound-medicine, and targetcompound-medicine networks were constructed using Cytoscape. Molecular docking was used to predict the predominant binding combination of core targets and components. We obtained 79 targets for angiogenesis; 41 targets were matched to 3839 compounds, of which 110 compounds were selected owing to their high correlation with angiogenesis. Fifty-five combinations in the network were obtained by molecular docking, among which PTGS2-astragalin $(-9.18 \mathrm{kcal} / \mathrm{mol}), \mathrm{KDR}$-astragalin $(-7.94 \mathrm{kcal} /$ $\mathrm{mol})$, PTGS2-quercetin $(-7.41 \mathrm{kcal} / \mathrm{mol})$, and PTGS2-myricetin $(-7.21 \mathrm{kcal} / \mathrm{mol})$ were top. These results indicated that the selected potential core compounds have good binding activity with the core targets. Eighty new combinations were obtained from the network, and the top combinations based on affinity were KDR-beta-carotene $(-10.13 \mathrm{kcal} / \mathrm{mol})$, MMP9-beta-sitosterol $(-8.04 \mathrm{kcal} / \mathrm{mol})$, MMP9-astragalin $(-7.82 \mathrm{kcal} / \mathrm{mol})$, and MMP9-diosgenin $(-7.51 \mathrm{kcal} / \mathrm{mol})$. The core targets included PTGS2, KDR, VEGFA, and MMP9. The essential components identified were astragalin, kaempferol, myricetin, quercetin, and $\beta$-sitosterol. The crucial Chinese medicines identified included Polygoni Cuspidati Rhizoma et Radix, Morus alba Root Bark, and Forsythiae Fructus. By systematically analysing the ingredients of traditional Chinese medicine and their targets, it is possible to determine their potential mechanisms of action against pathological angiogenesis. Our study provides a basis for further research and the development of new therapeutics for angiogenesis.

\section{Introduction}

John Hunter provided the first recorded scientific insights into angiogenesis and coined the term angiogenesis in 1787 [1]. Folkman [2-6] proposed the role of angiogenesis in tumour growth in 1971. He hypothesised that tumour growth depends on angiogenesis to increase blood supply and proposed stopping the blood supply from inhibiting tumour growth, which subsequently initiated the field of research on the relationship between angiogenesis and diseases. Angiogenesis is the process of capillary sprouting from preexisting vasculature, and it is highly induced by hypoxia and other biological processes $[5,7]$. The mechanisms underlying angiogenesis can be divided into two types. The first is sprouting angiogenesis, wherein vascular endothelial cell growth factor (VEGF) stimulates tip cells in 
the original blood vessel network to induce vascular sprouting $[8,9]$. The second is intussusceptive angiogenesis, which proceeds through transluminal tissue pillar formation and subsequent vascular splitting to the expansion and remodelling of microvascular networks [9-11].

Under normal circumstances, angiogenesis is a balance between inhibiting and growth factors. If the functions of either the inhibiting or growth factors are abnormal, it presents as overgrowth, defect, or malformation. Angiogenesis is essential for the growth and development of tumour cells. Under hypoxic conditions, tumours stimulate neovascularisation via the expression of growth factors such as VEGF [12]. Thus, exploring the inhibition of angiogenesis for the treatment of tumours has gained increased attention. However, some studies have shown that using antiangiogenic agents can induce potential resistance mechanisms such as autophagy, VEGF-dependent alterations, non-VEGF pathways, and stromal cell interactions [13-17]. Tumour cells may become accustomed to hypoxia or nutrient deprivation or induce angiogenesis via other growth factors $[18,19]$. Such events can lead to higher survival levels of the tumour cells. Moreover, some antiangiogenic agents can cause side effects such as acne-like rash, hypertension, and diarrhoea [20,21].

Traditional Chinese medicine is valuable for the treatment of various diseases, especially refractory diseases. More importantly, some studies proved that traditional Chinese medicine inhibits endothelial progenitor cells migration and tube formation without any cytotoxic activity [22]. Coadministration of traditional Chinese medicine and chemotherapy drugs could regulate angiogenesis, favour the delivery of chemotherapy drugs to the tumour lesion, promote apoptosis of tumour cells, enhance the effective treatment performance of chemotherapy drugs, and minimize the toxic side effects caused by chemotherapy drugs [23, 24]. Therefore, traditional Chinese medicine is more effective and safer in treating diseases. Previously, we found that many herbal extracts such as Epimedium brevicornu Maxim, Dalbergia odorifera T. Chen, and Trichosanthes kirilowii Maxim can regulate angiogenesis [25]. Cucurbitacin $\mathrm{E}$, a compound in herbal extracts, can inhibit tumour angiogenesis by inhibiting vascular endothelial growth factor receptor 2- (KDR/ VEGFR2-) mediated Jak-STAT3 and mitogen-activated protein kinase (MAPK) signalling pathways [26]. Astragaloside IV and curcumin can suppress the expression of fibroblast growth factor-2, matrix metalloproteinase 2, VEGF, hepatocyte growth factor, thrombosis-related factor tissue factor, and coagulation factor VII, thereby reducing the microvessel count [27]. The above herbal studies focused on single or several compounds related to angiogenesis. Nevertheless, during treatment, multiple herbal compounds interact or cross-react to regulate different targets and pathways. Thus, even though traditional Chinese medicine is safe for clinical treatment, the in vivo intervention mechanism is diverse, complex, and largely unknown.

Network pharmacology is a combination of pharmacology, biomedicine, systems biology, and network biology. Through various methods such as data mining, statistics, and modelling information visualization to recognize multicomponent synergy and assess each specific agent combinations' synergistic relationship [28-30], the mechanism of drugs in the biological network was analysed as well as determining which pathophysiological mechanisms are involved in the disease [28]. In this study, the angiogenesis targets were explored through network pharmacology, and the corresponding compounds and herbs were matched. Furthermore, the effect of herbal compounds on angiogenesis and the intervention mechanism was demonstrated. The findings of this study are expected to provide insights into the development of novel therapeutics for angiogenesis. The detailed workflow of the investigation is shown in Figure 1 .

\section{Materials and Methods}

2.1. Collection of Data regarding Angiogenesis-Related Targets. Data were collected from GeneCards (https://www. genecards.org/) [31], Therapeutic Target Database (TTD; http://db.idrblab.net/ttd/) [32], Online Mendelian Inheritance in Man (OMIM, https://omim.org) [33], DrugBank (http://www.drugbank.ca) [34], and DisGeNET (http:// www.disgenet.org) [35]. The keyword used to search these databases was "angiogenesis." After sorting and removing repeated targets, matching targets' full name was established using the UniProt database (https://www.uniprot.org/) [36].

2.2. Screening of Related Herbal Compounds. The targets were used to match the compounds related to angiogenesis in the Traditional Chinese Medicine Systems Pharmacology Database and Analysis Platform (TCMSP; http://tcmspw. com/) [37] and Encyclopedia of Traditional Chinese Medicine (ETCM; http://www.tcmip.cn/ETCM/index.php/ Home/Index/) [38]. The compounds and targets were imported to Cytoscape version 3.8.1 (Institute for Systems Biology, Seattle, WA, USA) [39] to construct a "targetcompound" network. After preliminary screening, the related compounds were verified through a literature review, and the compounds closely related to diseases were screened as related compounds.

\subsection{Collection of Related Traditional Chinese Medicines and Construction of a Target-Compound-Traditional Chinese Medicine Network. By collecting the traditional Chinese medicines related to compounds and constructing the "compound-traditional Chinese medicine" network com- bined with the "compound-target" network, the "target- compound-traditional Chinese medicine" network was constructed using Cytoscape 3.8.1 to explore and mine the relationships within the network. Key nodes were found by calculating the topological parameters of each node in the network using NetworkAnalyzer to preliminarily evaluate the effectiveness of traditional Chinese medicine and the compounds on angiogenesis.}

2.4. Statistics and Frequency Analysis of Traditional Chinese Medicine Information. Information related to traditional 


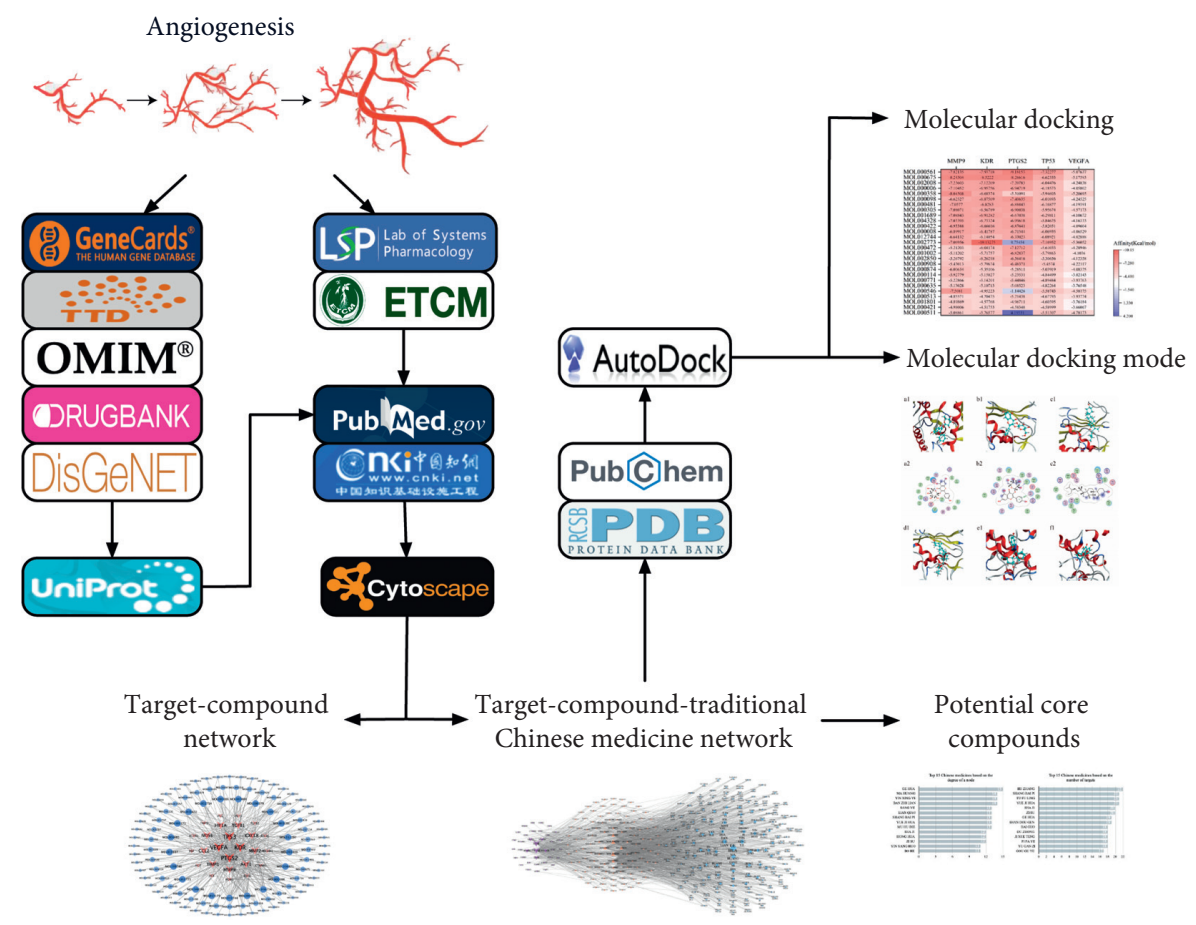

FIgURE 1: The workflow of the investigation.

Chinese medicine, including the four natures, five flavours, and meridians, was collected from the Chinese Pharmacopoeia (2020 edition) [40], the 13th five-year plan textbook of Traditional Chinese Medicine Pharmacy [41], and the Chinese Dictionary of Clinical Drugs [42]. If the information did not exist in these three documents, that medicine was excluded. IBM SPSS Statistics 26.0 was used to perform statistical tests and frequency analysis.

2.5. Molecular Docking for Targets and Compounds. To define the reliability of the interaction relationship between the core targets and core components in the "target-compoundtraditional Chinese medicine network" and explore the new drug-target combinations, the top five targets with a moderate value for target-compound-traditional Chinese medicine were selected as receptors. The crystal structures of these proteins were selected and preserved in PDB format from Biological Macromolecular Structures Enabling Breakthroughs in Research and Education (RCSB; http:// www.rcsb.org/pdb/). The 3D structures of the candidate compounds were downloaded and saved in SDF format from PubChem (https://pubchem.ncbi.nlm.nih.gov/). These SDF files were converted to the PDB format using Open Babel. The water molecules in the ligands were removed using AutoDock Tools 1.5.6 (Molecular Graphics Lab, La Jolla, CA, USA). After dispersing the ligands and receptors, nonpolar hydrogen bonds were added, and Gasteiger charges were calibrated and stored as pdbqt files. The selected potential core ligands were treated with energy minimisation, and the ligand atom type and calculated charge were saved in the pdbqt format. AutoDock Vina 1.1.2 [43] was used to calculate the docking score between the target and ligand to evaluate its matching degree and docking activity. A docking score of less than -4.25 indicated binding between the ligand and target. A score of less than -5.0 indicated better binding activity, and a score of less than -7.0 indicated vigorous docking activity. The ideal combinations were selected according to the affinity value, and the molecular docking pattern was displayed by MOE2019.

\section{Results}

3.1. Target Acquisition. We obtained 4609 targets related to angiogenesis from the GeneCards database. We identified the targets with strong correlation by calculating the median of their correlation coefficients because of the large number of targets. After six calculations, the medians were $0.65,1.39$, $3.25,4.96,7.47$, and 10.52 , and 74 targets with higher correlation coefficients were obtained. Furthermore, 5, 1, 0, and 0 related targets were separately obtained from the TTD, OMIM, DrugBank, and DisGeNET databases, respectively. After removing duplicate values and standardising them using the UniProt database, 79 angiogenesis targets were finally acquired. Containing the target information from TCMSP and ETCM databases were 49 and 4 target information, respectively. However, four were duplicate targets, and eight targets did not match any compound because the databases did not have information on related ingredients. Thus, only 41 targets were matched with small-molecule compounds and became potential targets. Table 1 shows the targets with over ten corresponding compounds.

A total of 3839 small-molecule compounds were matched with 41 potential targets to construct a targetcompound network consisting of 3440 nodes and 3839 
TABle 1: Target's information.

\begin{tabular}{lccc}
\hline Number & Gene & UniProt number & Protein \\
\hline 1 & PTGS2 & P35354 & Prostaglandin G/H synthase 2 \\
2 & KDR & P35968 & Vascular endothelial growth factor receptor 2 \\
3 & VEGFA & P15692 & Vascular endothelial growth factor A \\
4 & FGF1 & P05230 & Acidic fibroblast growth factor \\
5 & TP53 & P04637 & Cellular tumour antigen p53 \\
6 & MMP9 & P14780 & Matrix metalloproteinase-9 \\
7 & AKT1 & P31749 & RA kDa type IV collagenase \\
8 & MMP2 & P08253 & Interleukin-8 \\
9 & CXCL8 & P10145 \\
10 & FGF2 & P09038 & Basic fibroblast growth factor \\
11 & HIF1A & Q16665 & Hypoxia-inducible factor 1-alpha \\
12 & TGFB1 & P01137 & Transforming growth factor beta-1 \\
13 & CCL2 & P13500 & C-C motif chemokine 2 \\
14 & NOS3 & P29474 & Nitric oxide synthase, endothelial \\
\hline
\end{tabular}

Identification of candidate compounds and target-compound network construction.

edges. Although many small-molecule compounds showed a match, some were less related to the target or were associated with fewer studies. Hence, under the "Degree $>1$ " condition, the targets and compounds with a greater degree of interaction were screened out, resulting in 28 targets and 264 candidate compounds. These were then screened through a literature review for in vivo and in vitro activities. Finally, 110 compounds with research significance and 26 related targets were determined; these were used to construct the "target-compound" network (Figure 2), which contained 136 nodes and 370 edges.

\subsection{Identification of Traditional Chinese Medicines and} Target-Compound-Chinese Medicine Network Construction. A total of 447 Chinese medicines were obtained from 110 candidate compounds through database and literature matching. A compound-Chinese medicine network was first constructed according to the relationship between the compounds and Chinese medicines, and it contained 594 nodes and 2240 edges. Based on the connections of the node, the top nine Chinese medicines were Puerariae Flos (Ge Hua), Ephedra Herba (Ma Huang), Ginkgo Folium (Yin Xing Ye), Scutellariae Barbatae Herba (Ban Zhi Lian), Mori Folium (Sang Ye), Forsythiae Fructus (Lian Qiao), Morus alba Root Bark (Sang Bai Pi), Rosae Chinensis Flos (Yue Ji Hua), and Oroxyli Semen ( $\mathrm{Mu} \mathrm{Hu} \mathrm{Die),} \mathrm{which} \mathrm{contain} \mathrm{the}$ candidate compounds $15,14,14,14,13,13,13,13$, and 13, respectively. Through the bridging effect of candidate compounds, the targets of various Chinese medicines were obtained. The top six Chinese medicines were Polygoni Cuspidati Rhizoma et Radix (Hu Zhang), Morus alba Root Bark (Sang Bai Pi), Smilacis Glabrae Rhizoma (Tu Fu Ling), Rosae Chinensis Flos (Yue Ji Hua), Hippophae fructus (Sha $\mathrm{Ji}$ ), and Perilla frutescens ( $\mathrm{Zi} \mathrm{Su}$ ), which contain compounds $22,21,21,21,20$, and 20, respectively. Therefore, it can be inferred that these six Chinese medicines have a strong regulatory effect on the development of angiogenesis. Figure 3 shows the top 15 candidate compounds of traditional Chinese medicines based on the number of related targets and the degree of a node in the compound-Chinese medicine network. According to a previous study, the median compound degree value of the network was seven [44]. According to the three conditions of closeness centrality, betweenness centrality, and compound degree value greater than 20, there were 27 potential core compounds. The top five ingredients were quercetin, $\beta \beta$-sitosterol, kaempferol, luteolin, and ursolic acid. The remaining potential core compounds are shown in Table 2.

The target-compound-traditional Chinese medicine network was reconstructed by selecting traditional Chinese medicines with a degree $>4$. Their associated compounds and targets were organised to display the relationships among angiogenesis-related targets, compounds, and traditional Chinese medicines more intuitively (Figure 4).

3.3. Statistics and Frequency Analysis of Chinese Medicine Information. Information regarding flavour, natures, and meridian was collected and analysed for 413 traditional Chinese medicines. The highest frequencies of the four natures were warm and cold, accounting for $25.5 \%$ and $24.5 \%$, respectively. Flavour analysis revealed that the most predominant flavours were acrid and bitter, accounting for $28.9 \%$ and $27.6 \%$, respectively. The highest-ranked meridians related to angiogenesis were the liver, lungs, and stomach, accounting for $20.8 \%, 16.7 \%$, and $14.2 \%$, respectively. The results of the statistical analyses are shown in Table 3 and Figure 5.

3.4. Molecular Docking. The 27 core potential compounds were molecularly docked with five core targets, namely, matrix metallopeptidase 9 (MMP9), VEGFR2, prostaglandin-endoperoxide synthase 2 (PTGS2), TP53, and vascular endothelial growth factor A (VEGFA), and 135 sets of receptor-ligand docking results were obtained. Among the 135 receptor-ligand groups, 94 groups $(69.63 \%)$ showed affinity $<-5$, and 22 groups (16.30\%) showed affinity $<-7$.

Among the 135 combinations, 55 combinations were present in the target-compound network. Among these 55 combinations, the highest score for docking was observed for PTGS2-astragalin $(-9.18 \mathrm{kcal} / \mathrm{mol})$, and the lowest 


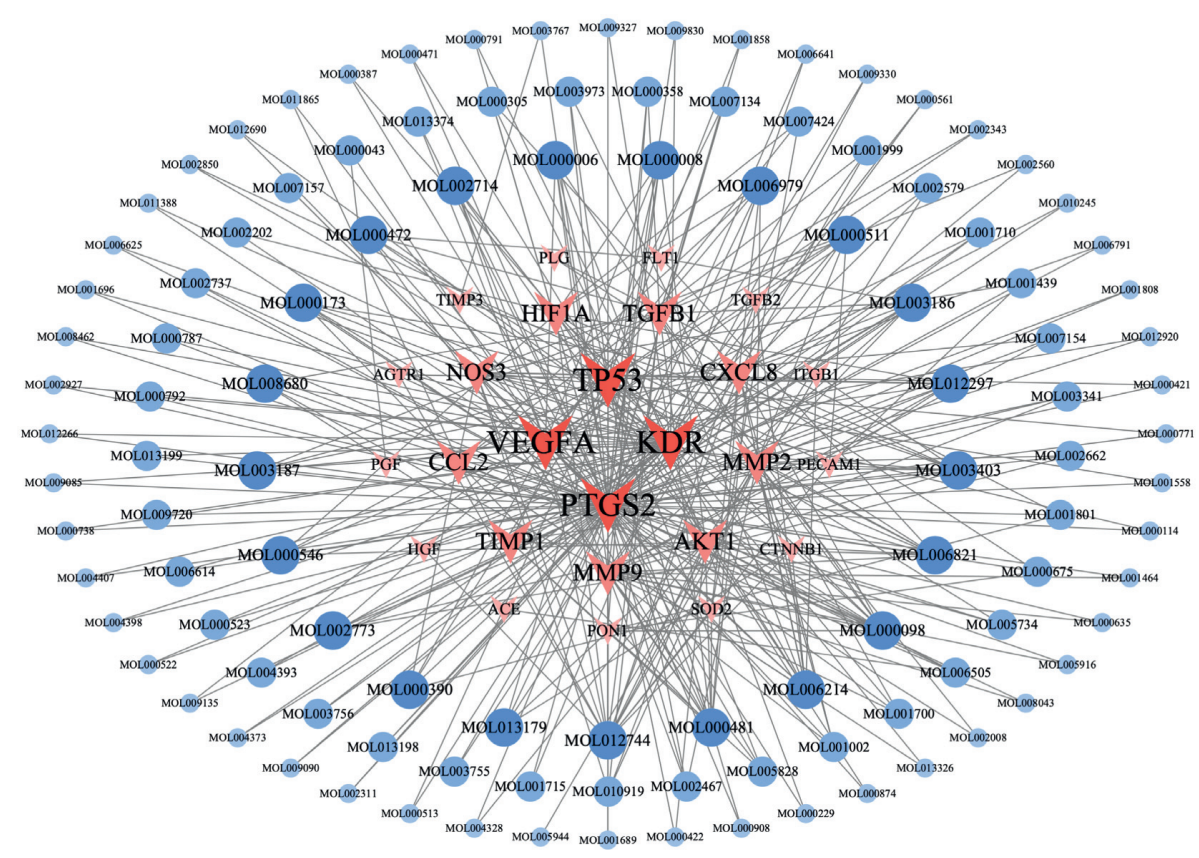

Figure 2: Target-compound network.
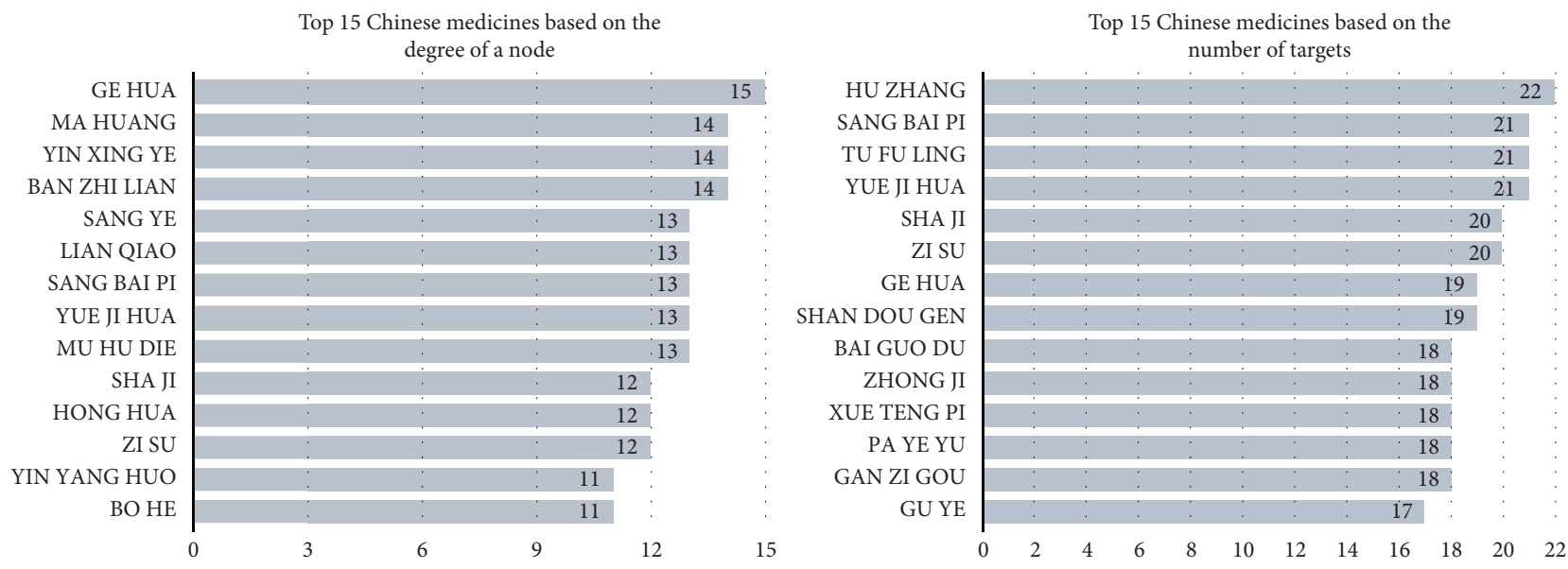

Figure 3: Number of candidate compounds and targets related to traditional Chinese medicines.

docking score was observed for PTGS2-ursolic acid $(4.20 \mathrm{kcal} / \mathrm{mol})$. The average of the above combinations was $-5.56 \mathrm{kcal} / \mathrm{mol}$. This result indicates that the screened potential core compounds may have better binding activity with the core target and supports the reliability of drugtarget interactions in the target-compound network to a certain extent.

Molecular docking results revealed 80 new combinations outside the target-compound network. The more ideal combinations outside the target-compound network were KDR-beta-carotene (-10.13 kcal/mol), MMP9-beta-sitosterol $(-8.04 \mathrm{kcal} / \mathrm{mol})$, MMP9-astragalin $(-7.82 \mathrm{kcal} / \mathrm{mol})$, and MMP9-diosgenin $(-7.51 \mathrm{kcal} / \mathrm{mol})$. There were 52 new combinations with affinity $<-5 \mathrm{kcal} / \mathrm{mol}$, suggesting that they all have good docking activity. The docking activity of these four combinations exceeded that of most combinations in the target-compound network; therefore, these are more likely to have a strong drug-target relationship. These docking results can provide data for the experimental screening and design of related Chinese medicines and ingredients in the future. The results are shown in Figure 6.

Considering the ideal combination of the affinity value of molecular docking and degree value of the targetcompound-drug network, nine more ideal combinations were selected. Their docking conditions are displayed in three-dimensional and two-dimensional molecular docking patterns. Each ligand was embedded in the active pocket of the target and interacted with multiple residues of the target through hydrophobic interaction and hydrogen bond formation (Figure 7). 
TABLE 2: Candidate compounds and targets information (degree $>20$ ).

\begin{tabular}{|c|c|c|c|c|c|}
\hline MolID & CAS & MolName & Degree & $\mathrm{OB}$ & $\mathrm{DL}$ \\
\hline MOL000098 & $117-39-5$ & Quercetin & 201 & 46.43 & 0.28 \\
\hline MOL000358 & $83-46-5$ & Beta-sitosterol & 192 & 36.91 & 0.75 \\
\hline MOL000422 & $520-18-3$ & Kaempferol & 136 & 41.88 & 0.24 \\
\hline MOL000675 & $112-80-1$ & Oleic acid & 120 & 33.13 & 0.14 \\
\hline MOL000006 & $491-70-3$ & Luteolin & 99 & 36.16 & 0.25 \\
\hline MOL000511 & $77-52-1$ & Ursolic acid & 88 & 16.77 & 0.75 \\
\hline MOL000008 & $520-36-5$ & Apigenin & 87 & 23.06 & 0.21 \\
\hline MOL000114 & $121-34-6$ & Vanillic acid & 86 & 35.47 & 0.04 \\
\hline MOL000305 & $143-07-7$ & Lauric acid & 69 & 23.59 & 0.04 \\
\hline MOL000513 & $149-91-7$ & 3,4,5-Trihydroxybenzoic acid (Gallic acid) & 57 & 31.69 & 0.04 \\
\hline MOL000908 & $515-13-9$ & Beta-elemene & 51 & 25.63 & 0.06 \\
\hline MOL000771 & $501-98-4$ & p-Coumaric acid & 48 & 43.29 & 0.04 \\
\hline MOL000635 & $121-33-5$ & Vanillin & 48 & 52.00 & 0.03 \\
\hline MOL000561 & $480-10-4$ & Astragalin & 39 & 14.03 & 0.74 \\
\hline MOL000472 & $518-82-1$ & Emodin & 37 & 24.40 & 0.24 \\
\hline MOL002850 & $128-37-0$ & Butylated hydroxytoluene & 35 & 40.0 & 0.07 \\
\hline MOL002773 & $7235-40-7$ & Beta-carotene & 34 & 37.18 & 0.58 \\
\hline MOL001801 & $69-72-7$ & Salicylic acid & 30 & 32.13 & 0.03 \\
\hline MOL000874 & $552-41-0$ & Paeonol & 30 & 28.79 & 0.04 \\
\hline MOL000481 & $446-72-0$ & Genistein & 26 & 17.93 & 0.21 \\
\hline MOL000421 & $59-67-6$ & Nicotinic acid & 25 & 47.65 & 0.02 \\
\hline MOL002008 & $529-44-2$ & Myricetin & 25 & 13.75 & 0.31 \\
\hline MOL004328 & $67604-48-2$ & Naringenin & 24 & 59.29 & 0.21 \\
\hline MOL001689 & $480-44-4$ & Acacetin & 23 & 34.97 & 0.24 \\
\hline MOL012744 & $501-36-0$ & Resveratrol & 23 & 19.07 & 0.11 \\
\hline MOL001002 & $476-66-4$ & Ellagic acid & 21 & 43.06 & 0.43 \\
\hline MOL000546 & $512-04-9$ & Diosgenin & 20 & 80.88 & 0.81 \\
\hline
\end{tabular}

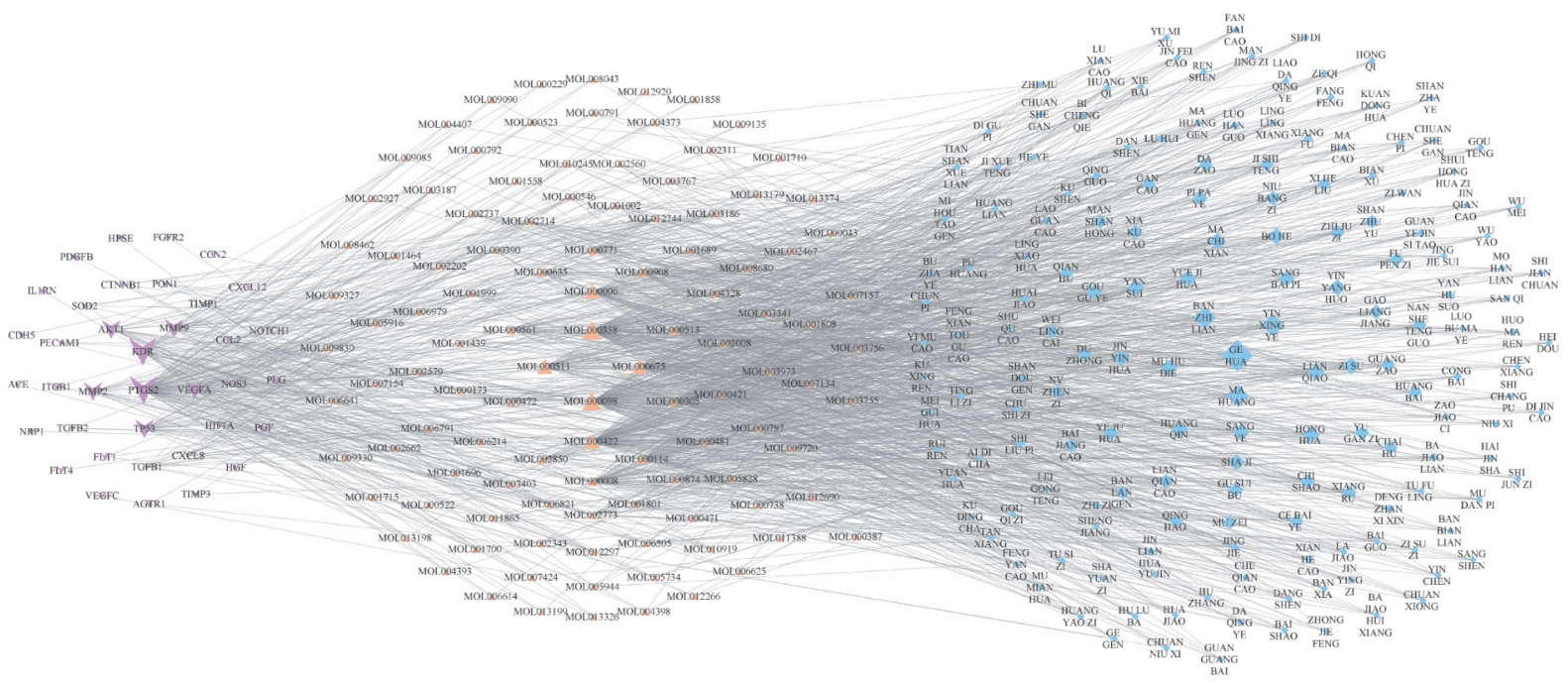

Figure 4: Target-compound-traditional Chinese medicine network. The diamond-shaped nodes represent Chinese medicine, the triangular nodes represent the ingredients, and the $\mathrm{V}$-shaped nodes represent the target. The icon size of each node is positively correlated with its degree value.

\section{Discussion}

Angiogenesis is a complex process that requires the coordinated regulation of several activating and inhibitory pathways. It participates in developing many diseases, such as cancers, atherosclerosis, rheumatoid arthritis, hepatitis, and inflammation. There are many factors involved in the regulation of angiogenesis. As traditional Chinese medicines, which have a curative effect in clinical treatment, are applied in combination, they contain multiple components and targets. Therefore, elucidating the mechanism and exploring the potential components of traditional Chinese medicines are of great significance in developing novel drugs. 
TABLE 3: Natures and flavours of traditional Chinese medicine regulating angiogenesis.

\begin{tabular}{|c|c|c|c|c|c|}
\hline Four natures & Frequency & Percentage & Five flavours & Frequency & Percentage \\
\hline Warm & 103 & 25.5 & Acrid & 160 & 28.9 \\
\hline Cold & 99 & 24.5 & Bitter & 153 & 27.6 \\
\hline Neutral & 84 & 20.8 & Sweet & 139 & 25.1 \\
\hline Slightly cold & 49 & 12.1 & Sour & 27 & 4.9 \\
\hline Cool & 34 & 8.4 & Astringent & 25 & 4.5 \\
\hline Slightly warm & 23 & 5.7 & Slightly bitter & 22 & 4 \\
\hline Hot & 10 & 2.5 & Salty & 12 & 2.2 \\
\hline Great cold & 1 & 0.2 & Bland & 9 & 1.6 \\
\hline \multirow[t]{3}{*}{ Great hot } & 1 & 0.2 & Slightly acrid & 3 & 0.5 \\
\hline & & & Slightly sweet & 3 & 0.5 \\
\hline & & & Slightly sour & 1 & 0.2 \\
\hline
\end{tabular}

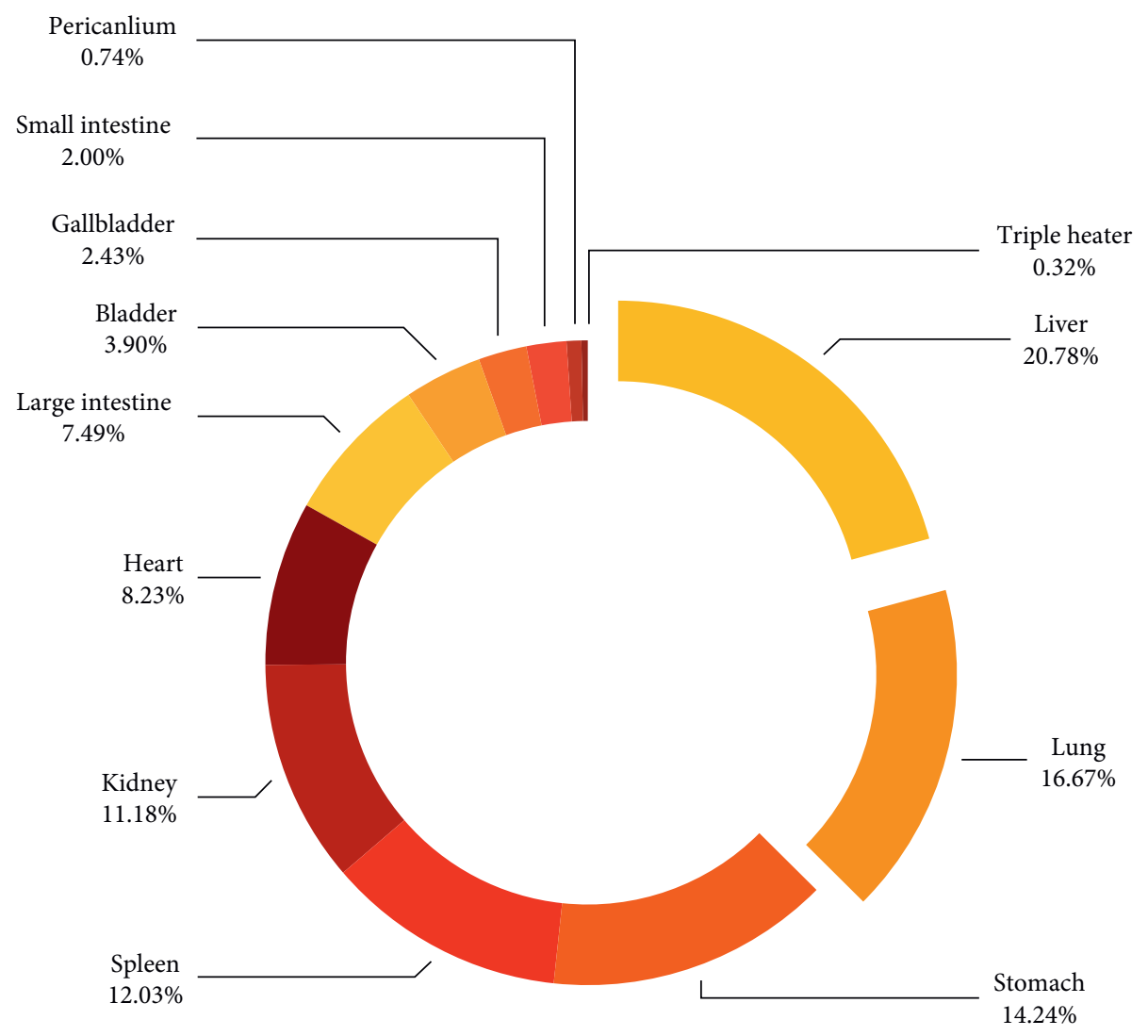

FIGURE 5: Channel entry of traditional Chinese medicine regulating angiogenesis.

4.1. Target. According to the target-compound network screening results, the top scores were obtained for PTGS2, KDR, VEGFA, and MMP9. The primary role of PTGS2 in angiogenesis is to induce the synthesis of individual prostanoids such as PGD2, PGE2, PGF2a, PGI2, and TXA2. Prostaglandin (PG) can boost VEGF production in a paracrine, intracrine, or autocrine manner. Moreover, VEGF stimulates PTGS2 expression, thereby triggering PG production. This, in turn, increases the levels of PGs and stimulates the expression of angiogenic factors such as VEGF and bFGF [45]. VEGF expression is regulated by many factors such as epidermal growth factor, hypoxiainducible factor (HIF), and platelet-derived growth factor
(PDGF). During angiogenesis, VEGF signalling regulates the activities of several kinases through VEGFR2 and guides the proliferation, migration, and survival of cells.

An increased number of endothelial cells, both tip and stalk cells, is a significant feature of vascular proliferation. Endothelial tip cells are induced by VEGF gradients and promote the formation of filopodia. The molecular regulation of these events occurs via the activation of Notch signalling and increased expression of Notch ligands on endothelial cells. A high level of Notch signalling can decrease VEGFR2 expression. Physiological homeostasis requires this negative feedback loop [46]. One crucial event implicated in the migration and proliferation of vascular 


\begin{tabular}{|c|c|c|c|c|c|c|}
\hline & $\begin{array}{c}\text { MMP9 } \\
\end{array}$ & $\begin{array}{c}\text { KDR } \\
1\end{array}$ & $\begin{array}{c}\text { PTGS2 } \\
\end{array}$ & $\begin{array}{c}\text { TP53 } \\
1 \\
\end{array}$ & $\begin{array}{c}\text { VEGFA } \\
1 \\
\end{array}$ & \multirow{7}{*}{$\begin{array}{l}\text { Affinity }(\mathrm{kcal} / \mathrm{mol}) \\
\\
\square-10.15\end{array}$} \\
\hline MOL000561 - & -7.82135 & -7.93718 & -9.18153 & -7.32277 & -5.07637 & \\
\hline MOL000675 - & -8.24304 & -8.5222 & -8.26616 & -6.62333 & -5.17593 & \\
\hline MOL002008 & -7.23603 & -7.12269 & -7.20783 & -6.04476 & -4.24838 & \\
\hline MOL000006 - & -7.10452 & -6.95756 & -6.94719 & -6.18573 & -4.05802 & \\
\hline MOL000358 & -8.04308 & -6.68374 & -5.31091 & -5.94603 & -5.20695 & \\
\hline MOL000098 & -6.62527 & -6.87569 & -7.40635 & -6.01093 & -4.24525 & \\
\hline MOL000481 - & -7.0577 & -6.8283 & -6.88843 & -6.16877 & -4.19391 & \multirow{6}{*}{--7.280} \\
\hline MOL000305 & -7.08071 & -6.56789 & -6.90038 & -5.95674 & -4.57173 & \\
\hline MOL001689 & -7.08843 & -6.91282 & -6.67038 & -6.29811 & -4.10672 & \\
\hline MOL004328 - & -7.03395 & -6.73324 & -6.99618 & -5.84675 & -4.16333 & \\
\hline MOL000422 & -6.93388 & -6.66616 & -6.87641 & -5.82051 & -4.09604 & \\
\hline MOL000008 & -6.89917 & -6.41787 & -6.71544 & -6.06935 & -4.06529 & \\
\hline MOL012744 & -6.64132 & -6.14054 & -6.33023 & -6.08921 & -4.02888 & \multirow{3}{*}{--4.410} \\
\hline MOL002773 & -7.06956 & -10.13275 & 0.75454 & -7.16952 & -5.36052 & \\
\hline MOL000472 - & -5.21203 & -6.04174 & -7.12712 & -5.61033 & -4.28946 & \\
\hline MOL001002 & -5.11202 & -5.71757 & -6.82837 & -5.79863 & -4.1036 & \multirow[t]{3}{*}{--1.540} \\
\hline MOL002850 & -5.26792 & -6.26218 & -6.56416 & -5.30656 & -4.12358 & \\
\hline MOL000908 - & -5.43013 & -5.79674 & -6.48371 & -5.4574 & -4.22117 & \\
\hline MOL000874 - & -6.00634 & -5.35106 & -5.28511 & -5.03919 & -4.08375 & \multirow[t]{3}{*}{--1.330} \\
\hline MOL000114 & -5.92779 & -5.15027 & -5.23531 & -4.84499 & -3.82143 & \\
\hline MOL000771 - & -5.22866 & -5.14201 & -5.44846 & -4.89484 & -3.93763 & \\
\hline MOL000635 & -5.13028 & -5.10713 & -5.08523 & -4.82264 & -3.76548 & \multirow{6}{*}{-4.200} \\
\hline MOL000546 - & -7.5081 & -4.95223 & -1.14426 & -5.58743 & -4.58175 & \\
\hline MOL000513 - & -4.85571 & -4.70475 & -5.21438 & -4.67793 & -3.93774 & \\
\hline MOL001801 - & -4.89869 & -4.57768 & -4.96711 & -4.60395 & -3.76184 & \\
\hline MOL000421 - & -4.98006 & -4.51753 & -4.58348 & -4.58999 & -3.66067 & \\
\hline MOL000511 - & -5.08861 & -5.76577 & 4.19531 & -5.51307 & -4.78173 & \\
\hline
\end{tabular}

Figure 6: Molecular docking results.

endothelial cells is the proteolytic degradation of basement membranes and extracellular matrix (ECM) components by matrix metalloproteinases (MMPs) [47]. The secretion of MMPs allows endothelial cells to penetrate their underlying basement membrane and eliminate the contact inhibition that blocks endothelial cell proliferation [48]. The gene expression of MMPs may stimulate the production and secretion of major proangiogenic factors such as VEGF and fibroblast growth factor-2, which promote angiogenesis [48-50]. MMP9 cleaves ECM proteins and activates cytokines and chemokines to regulate tissue remodelling [51]. In the intracardiac injection experiment, the injected ECMderived substance promoted cell attachment, migration, and proliferation, induced extracellular signal-regulated kinase (ERK) $1 / 2$ activation, and promoted arteriogenesis [50]. In summary, the above targets play an essential role in regulating angiogenesis, and they are the preferred targets for traditional Chinese medicine intervention in angiogenesis.

4.2. Ingredients. Molecular docking showed that the components that bind well to the targets are astragaloside, kaempferol, myricetin, quercetin, and $\beta$-sitosterol. Astragalin suppresses interleukin-1 $\beta$ - (IL-1 $\beta$-) induced inflammatory mediators by activating peroxisome proliferator-activated receptor- $\gamma$, which subsequently inhibits IL- $1 \beta$-induced nuclear factor- (NF-) $\kappa \mathrm{B}$ and MAPK activation [52]. NF$\kappa \mathrm{B}$ subunit p65 activates the transcription of HIF- $1 \alpha$ and its target gene VEGF-A. Regulating HIF- $1 \alpha$ via NF- $\kappa \mathrm{B}$ activation can contribute to angiogenesis [53]. Kaempferol is an antioxidant that reduces reactive oxygen species (ROS) metabolism by inhibiting the NF- $\kappa \mathrm{B}$ pathway and upregulation of the associated transcriptional pathway [54]. ROS regulate angiogenesis via two different mechanisms: the HIF-VEGF/VEGFR2 signalling pathway and the VEGFindependent mechanism involving the generation of lipid oxidation products [55]. Endothelial nitric oxide synthase (eNOS) plays an essential role in regulating cell migration activities and vascular permeability [56]. Myricetin and quercetin inhibit thioredoxin reductase (TrxR) in an NADPH- and concentration-dependent manner [57]. TrxR is a part of the thioredoxin (Trx) system, including Trx and $\mathrm{NADPH}$ [58]. This system plays essential roles in regulating cellular redox signalling and contributes to the regulation of VEGF-mediated signalling [59-61]. For example, Trx1, in endothelial cells, prevents von Hippel-Lindau-mediated degradation of the transcription factor HIF1, leading to the induction of VEGF expression [62]. The action mechanism of myricetin and quercetin in angiogenesis is not yet well understood, but it may be related to the Trx system. $\beta$-sitosterol administration was reported to reduce the expression of chemokines and the activity of MMP2 and MMP9 [63]. In summary, the above components of traditional Chinese medicine can be investigated for intervention in angiogenesis.

4.3. Chinese Medicine. An experiment using umbilical vein endothelial cells demonstrated that angiogenesis could be regulated by the extract of Polygoni Cuspidati Rhizoma et Radix via the inhibition of the phosphorylation of downstream signalling molecules such as ERK, Akt, and eNOS by VEGF/VEGFR2 [64]. These molecules can regulate endothelial cell survival, proliferation, and migration $[65,66]$. The 


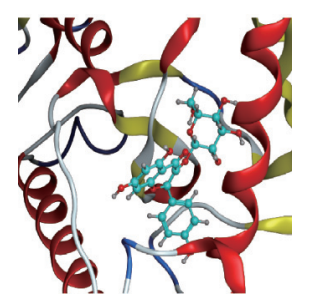

a1

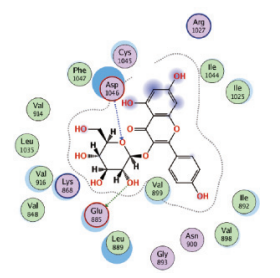

a2

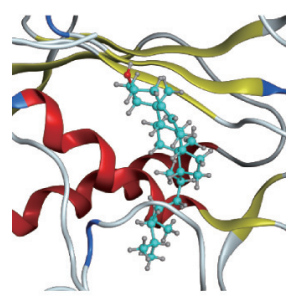

d1

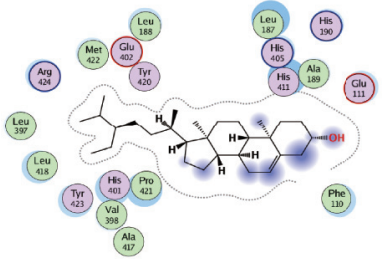

$\mathrm{d} 2$

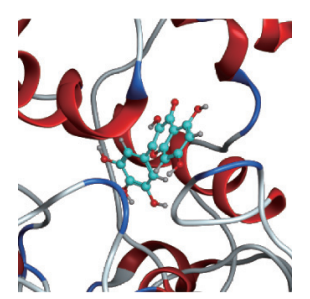

g1

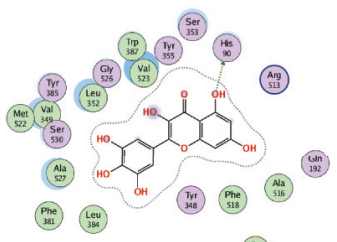

$\left(\begin{array}{ccc}\text { III } \\ \text { siz }\end{array}\right.$

g2

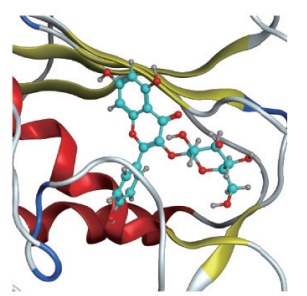

b1

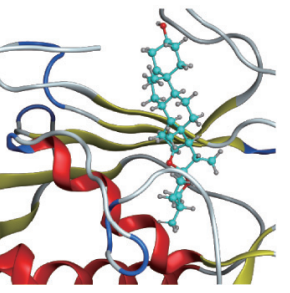

c1

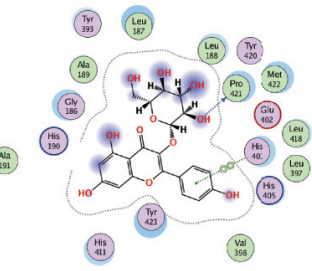

b2

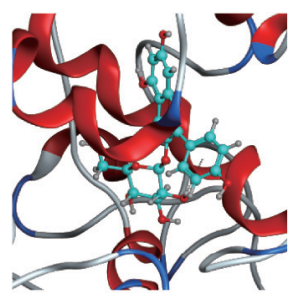

e1

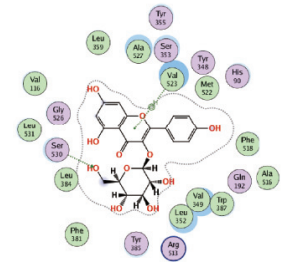

e2

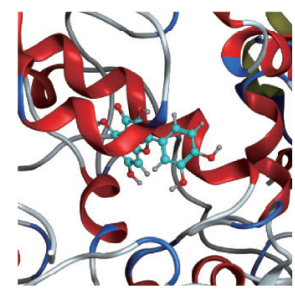

h1

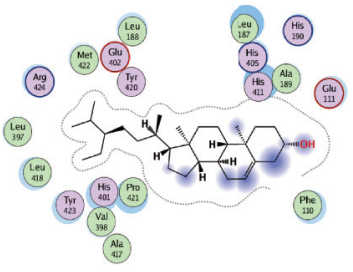

c2

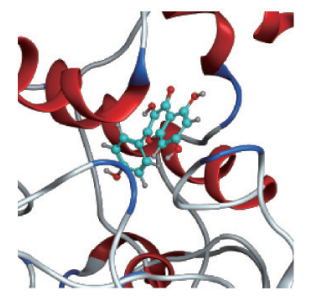

f1

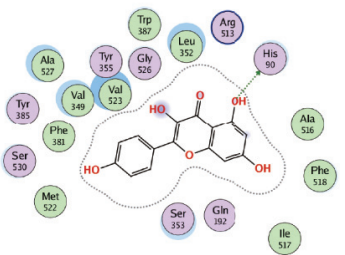

f2

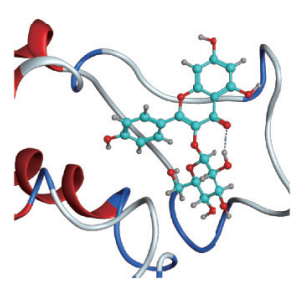

i1

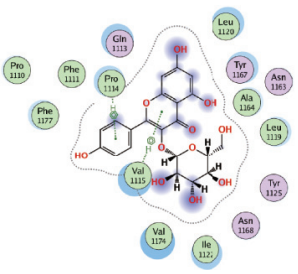

i2

FIGURE 7: Molecular docking model. In the 3D structure of ligand-protein complexes, the protein backbone is represented as a cartoon ( $\alpha$-helices in red and $\beta \beta$-sheets in green), peptide chains are coloured differently, and ligands are displayed in blue. The $2 \mathrm{D}$ interaction model shows amino acids circled differently according to their propensity for water; green: hydrophobic residues; purple: polar residues. (a) KDR6gqq-astragalin; (b) MMP9-1gkc-astragalin; (c) MMP9-1gkc-beta-diosgenin; (d) MMP9-1gkc-beta-sitosterol; (e) PTGS2-5ikq-astragalin; (f) PTGS2-5ikq-kaempferol; (g) PTGS2-5ikq-myricetin; (h) PTGS2-5ikq-quercetin; (i) tp53-1jsp-astragalin. 
extract of Morus alba Root Bark inhibits the proliferation and migration of vascular smooth muscle cells induced by PDGF. It stimulates the formation of nitric oxide (NO) in endothelial cells [67]. $\mathrm{NO}$ is a vital gaseous signalling molecule that participates in the growth and remodelling of essential biochemical and molecular reactions necessary for regulating angiogenesis. The $\mathrm{NO}$-induced activation of soluble guanylate cyclase increases cyclic guanosine monophosphate formation and protein kinase G activity to modulate signalling cascades by phosphorylation MAPKs, which successively phosphorylate and activate downstream proteins such as ERK1/2 $[68,69]$. These events regulate the proliferation and migration of endothelial cells, resulting in angiogenesis. Forsythiae Fructus aqueous extract triggers the inhibition of oxidative stress and inflammation via the MAPKs/Nrf2/HO-1 signalling pathway and inhibits cancer cell proliferation and angiogenesis [70].

Traditional Chinese medicines can be classified based on the four natures, five flavours, and meridians. Information about these factors can be related to efficacy and utility in a clinical setting [71]. In this study, the most prevalent natures were found to be warm and cold. According to Chapter 74 of the Zhizhenyao Dalunpian: Discussion on the Most Important and Abstruse Theory - Plain Questions, which describes the rules of using traditional Chinese medicine in treatment, diseases caused by cold are treated with warmnatured therapy, and heat syndromes are treated with coldnatured therapy.

The core function of warm is to activate blood, replenish qi, and prevent the inhibition of water. It is usually used in treating yang deficiency or problems in fluid transformation such as heart failure. In some studies, it is shown that the extract of the warm-natured drugs such as Allicin [72] and astragaloside IV [73], through regulating sarcoplasmic reticulum $\mathrm{Ca} 2+$ pump, improved the distribution and expression of PECAM-1, enhanced the migration and angiogenesis ability of cardiac microvascular endothelial cells, promoted angiogenesis, protected myocardial ischemia, inhibited cardiac hypertrophy and fibrosis, and reduced myocardial cell apoptosis. The primary function of cold is heat-clearing, detoxicating, and draining fire. It inhibited the release of inflammatory factors to treat the diseases caused by inflammation [74] such as cancer. Some extracts of the cold-natured drugs such as tetrandrine [75], artemisinin [76], and andrographolide [77] inhibited endothelial cell proliferation, adhesion, invasion, and tube formation by targeting vascular endothelial growth factor and blocking angiogenesis and invasion of cancer cells

Moreover, the highest-ranked flavours were acrid, bitter, and sweet. Acrid promotes diffusion and outthrust with dissipation-it is favourable for treating blood-stasis, blockage, and accumulations, such as ischemic stroke and cancer. Galangin and tetramethylpyrazine were extracted and isolated from traditional Chinese medicine with acrid flavours. Galangin ameliorated neurological scores, cerebral infarct volume, and cerebral edema through improving the neurovascular microenvironment [78]. Tetramethylpyrazine restrains angiogenesis by suppressing the ERK1/2 and Akt pathways and promotes apoptosis of tumour cells [24].
Furthermore, the bitter flavour and cold-natured drugs exerted a similar effect on draining fire, but the bitterness was better to dry dampness and drain fire downward. It also has an excellent therapeutic effect on diseases caused by inflammation. The primary function of sweet is to supplement and harmonize the centre and relax tension. It is appropriate for patients who are in pain and asthenia. Under the Chinese medicine theory, diabetic retinopathy was often classified as the pattern of dual vacuity of qi and yin and was influential in treating diabetic retinopathy by boosting qi and nourishing, clearing heat, and breeding body fluids [79]. Plantaginis semen belongs to the extract of sweet medicinals, which ameliorated diabetic retinopathy through increasing vascular permeability and retinal vessel diameter and restrained retinal vascular dilation [80].

The theory of channel tropism posits that medicines have selective therapeutic effects in different Zang-fu organs. This study shows that the top two meridians related to the studied traditional medicines were the liver and lungs. The liver is a crucial organ that regulates blood, including its storage, filtration, and bleeding. In contrast, the lungs control the qi and are intimately related to the qi of the chest. They also play an essential role in the blood and qi movement in circulation in the body. Thus, the liver and lungs closely collaborate in regulating the qi and blood. Because of the above, it can be presumed that impaired angiogenesis, according to the basic theory of Chinese medicine, is associated with the failure of the liver and lungs to perform their functions. Qi stagnation and blood stasis cause poor circulation of blood, which leads to the collection of waterhumour, with the stagnant qi being transformed into heat. Therefore, traditional Chinese medicine's fundamental treatment principles are to warm the yang, rectify the qi, and activate the blood. In cases of long-time ailment, efforts should be made to eliminate dampness and clear heat.

\section{Conclusions}

In this study, we used network pharmacology to identify proteins related to angiogenesis through databases and documentation. Also, we constructed a target-compoundtraditional Chinese medicine network, which was explored and analysed for the potential compounds and mechanisms of traditional Chinese medicine that participated in angiogenesis. The findings of this study can effectively narrow the scope of screening, improve scientific research efficiency, and reduce economic costs in the research of therapeutic agents to treat angiogenesis. However, this study is preliminary and is based on database analysis; therefore, it does not fully demonstrate the actual situation or verify the participation of traditional Chinese medicine in angiogenesis in vivo. The specific molecular mechanism still needs to be explored through subsequent experimental research.

\section{Abbreviations}

ECM: Extracellular matrix

ETCM: Encyclopedia of Traditional Chinese Medicine

HIF: Hypoxia-inducible factor 
OMIM: Online Mendelian Inheritance in Man

PDGF: Platelet-derived growth factor

ROS: Reactive oxygen species

TTD: Therapeutic Target Database

VEGF: Vascular endothelial cell growth factor.

\section{Data Availability}

The data and materials used for this study will be made available by the corresponding author Qingyong He upon reasonable request.

\section{Disclosure}

Wenchao Dan is the co-first author. An earlier version of this paper has been presented as a preprint in "Research Square" for scholars to comment on (https://www. researchsquare.com/article/rs-104137/v1). The funders had no role in the study design, data collection and analysis, decision to publish, or preparation of the manuscript.

\section{Conflicts of Interest}

The authors declare that they have no conflicts of interest.

\section{Authors' Contributions}

Wingyan Yun, Jinlei Liu, Xinyuan Guo, and Min Li collected the related targets of angiogenesis and related molecular compounds. Wingyan Yun and Wenchao Dan performed the network pharmacology analysis and molecular docking, and Wingyan Yun wrote the manuscript. Wenchao Dan and Qingyong $\mathrm{He}$ revised the manuscript. All authors were responsible for reviewing data and read and approved the final manuscript.

\section{Acknowledgments}

The authors gratefully acknowledge Jianliang $\mathrm{Li}$ and Jianbo Guo (Beijing University of Chinese Medicine) for the study design and overall guidance. This study was supported by the Beijing Science and Technology Rising Star (Z181100006218035), the National Natural Science Foundation of China (81202803), and the National Administration of TCM Project Fund (G2T-FJS- 2019-203).

\section{References}

[1] P. Lenzi, G. Bocci, and G. Natale, John Hunter and the origin of the term "angiogenesis", 2016.

[2] J. Folkman, "Tumor Angiogenesis: therapeutic Implications," New England Journal of Medicine, vol. 285, no. 21, pp. 1182-1186, 1971.

[3] M. A. Gimbrone, S. B. Leapman, R. S. Cotran, and J. Folkman, "Tumor dormancy in vivo by prevention of neovascularization," The Journal of Experimental Medicine, vol. 136, no. 2, pp. 261-276, 1972.

[4] D. Ribatti, "Judah Folkman, a pioneer in the study of angiogenesis," Angiogenesis, vol. 11, no. 1, pp. 3-10, 2008.

[5] J. Folkman, Angiogenesis In Biology Of Endothelial Cells, pp. 412-428, Springer, Heidelberg, Germany, 1984.
[6] J. Folkman and C. Haudenschild, "Angiogenesis in vitro," Nature, vol. 288, no. 5791, pp. 551-556, 1980.

[7] N. Warmke, A. M. N. Walker, and R. M. Cubbon, "Angiogenesis," Encyclopedia of Cardiovascular Research and Medicine, pp. 85-96, 2018.

[8] F. Hillen and A. W. Griffioen, "Tumour vascularization: sprouting angiogenesis and beyond," Cancer and Metastasis Reviews, vol. 26, pp. 3-4, 2007.

[9] W. Risau, "Mechanisms of angiogenesis," Nature, vol. 386, no. 6626, pp. 671-674, 1997.

[10] S. J. Mentzer and M. A. Konerding, "Intussusceptive angiogenesis: expansion and remodeling of microvascular networks," Angiogenesis, vol. 17, no. 3, pp. 499-509, 2014.

[11] A. N. Makanya, R. Hlushchuk, and V. G. Djonov, "Intussusceptive angiogenesis and its role in vascular morphogenesis, patterning, and remodeling," Angiogenesis, vol. 12, no. 2, pp. 113-123, 2009.

[12] S. Krishna Priya, R. P. Nagare, V. S. Sneha et al., "Tumour angiogenesis-Origin of blood vessels," International Journal of Cancer, vol. 139, no. 4, pp. 729-735, 2016.

[13] F. Shojaei and N. Ferrara, "Role of the microenvironment in tumor growth and in refractoriness/resistance to antiangiogenic therapies," Drug Resistance Updates, vol. 11, no. 6, pp. 219-230, 2019.

[14] F. Shojaei, "Anti-angiogenesis therapy in cancer: current challenges and future perspectives," Cancer Letters, vol. 320, no. 2, pp. 130-137, 2018.

[15] E. A. Kuczynski and A. R. Reynolds, "Vessel co-option and resistance to anti-angiogenic therapy," Angiogenesis, vol. 23, no. 1, pp. 55-74, 2019.

[16] Y. Haibe, M. Kreidieh, H. El Hajj et al., "Resistance mechanisms to anti-angiogenic therapies in cancer," Frontiers in Oncology, vol. 10221 pages, 2020.

[17] A. Chandra, J. Rick, G. Yagnik, and M. K. Aghi, Autophagy as a Mechanism for Anti-angiogenic Therapy Resistance Seminars In Cancer Biology, Elsevier, Amsterdam, Netherlands, 2019.

[18] Y. Crawford and N. Ferrara, "Tumor and stromal pathways mediating refractoriness/resistance to anti-angiogenic therapies," Trends in Pharmacological Sciences, vol. 30, no. 12, pp. 624-630, 2009.

[19] R. Ronca, M. Benkheil, S. Mitola, S. Struyf, and S. Liekens, "Tumor angiogenesis revisited: regulators and clinical implications," Medicinal Research Reviews, vol. 37, no. 6, pp. 1231-1274, 2017.

[20] C. Viallard and B. Larrivée, "Tumor angiogenesis and vascular normalization: alternative therapeutic targets," Angiogenesis, vol. 20, no. 4, pp. 409-426, 2018.

[21] C. Widakowich, G. De Castro, E. De Azambuja, P. Dinh, and A. Awada, "Side effects of approved molecular targeted therapies in solid cancers," The oncologist, vol. 12, no. 12, pp. 1443-1455, 2013.

[22] H.-P. Lee, Y.-C. Liu, P.-C. Chen et al., "Tanshinone IIA inhibits angiogenesis in human endothelial progenitor cells in vitro and in vivo," Oncotarget, vol. 8, no. 65, Article ID 109217, 2017.

[23] L.-Q. Yang, R.-Y. Li, X.-Y. Yang et al., "Co-administration of shexiang baoxin pill and chemotherapy drugs potentiated cancer therapy by vascular-promoting strategy," Frontiers in Pharmacology, vol. 10, p. 565, 2019.

[24] L. Zou, X. Liu, J. Li et al., "Tetramethylpyrazine enhances the antitumor effect of paclitaxel by inhibiting angiogenesis and inducing apoptosis," Frontiers in Pharmacology, vol. 10707 pages, 2019. 
[25] S. Wang, Z. Zheng, Y. Weng et al., "Angiogenesis and antiangiogenesis activity of Chinese medicinal herbal extracts," Life sciences, vol. 74, no. 20, pp. 2467-2478, 2004.

[26] Y. Dong, B. Lu, X. Zhang et al., "Cucurbitacin E, a tetracyclic triterpenes compound from Chinese medicine, inhibits tumor angiogenesis through VEGFR2-mediated Jak2-STAT3 signaling pathway," Carcinogenesis, vol. 31, no. 12, pp. 20972104, 2015.

[27] S. Zhang, D. Tang, W. Zang et al., "Synergistic inhibitory effect of traditional Chinese medicine astragaloside IV and curcumin on tumor growth and angiogenesis in an orthotopic nude-mouse model of human hepatocellular carcinoma," Anticancer Research, vol. 37, no. 2, pp. 465-474, 2017.

[28] L. Shao and B. Zhang, "Traditional Chinese medicine network pharmacology: theory, methodology and application," Chinese Journal of Natural Medicines, vol. 11, no. 2, pp. 110-120, 2013.

[29] S. Li, B. Zhang, and N. Zhang, "Network target for screening synergistic drug combinations with application to traditional Chinese medicine," BMC Systems Biology, vol. 5, no. 1, pp. 1-13, 2013.

[30] G.-B. Zhang, Q.-Y. Li, Q.-L. Chen, and S.-B. Su, "Network pharmacology: a new approach for Chinese herbal medicine research," Evidence-Based Complementary and Alternative Medicine, vol. 2013, Article ID 621423, 9 pages, 2013.

[31] M. Safran, I. Dalah, J. Alexander et al., "GeneCards Version 3: the human gene integrator," Database, vol. 2010, 2010.

[32] Y. Wang, S. Zhang, F. Li et al., "Therapeutic target database: enriched resource for facilitating research and early development of targeted therapeutics," Nucleic Acids Research, vol. 48, pp. D1031-D1041, 2020.

[33] J. S. Amberger, C. A. Bocchini, F. Schiettecatte, A. F. Scott, and A. Hamosh, "OMIM. org: Online Mendelian Inheritance in Man (OMIM $\left.{ }^{\circledR}\right)$, an online catalog of human genes and genetic disorders," Nucleic Acids Research, vol. 43, pp. D789-D798, 2020.

[34] D. S. Wishart, Y. D. Feunang, A. C. Guo et al., "DrugBank 5.0: a major update to the DrugBank database," Nucleic Acids Research, vol. 46, pp. D1074-D1082, 2018.

[35] J. Piñero, J. M. Ramírez-Anguita, J. Saüch-Pitarch et al., "The DisGeNET knowledge platform for disease genomics update," Nucleic Acids Research, vol. 48, pp. D845-D855, 2019.

[36] U. Consortium, "UniProt: the universal protein knowledgebase," Nucleic Acids Research, vol. 46, no. 5, p. 2699, 2017.

[37] J. Ru, P. Li, J. Wang et al., "TCMSP: a database of systems pharmacology for drug discovery from herbal medicines," Journal of Cheminformatics, vol. 6, no. 1, p. 13, 2014.

[38] H.-Y. Xu, Y.-Q. Zhang, Z.-M. Liu et al., "ETCM: an encyclopaedia of traditional Chinese medicine," Nucleic Acids Research, vol. 47, pp. D976-D982, 2019.

[39] P. Shannon, A. Markiel, O. Ozier et al., "Cytoscape: a software environment for integrated models of biomolecular interaction networks," Genome Research, vol. 13, no. 11, pp. 24982504, 2003.

[40] C. P. Commission, Chinese Pharmacopoeia (2020 Edition), China Medical Science Press, Beijing, China, 2020.

[41] Z.-X. Zhou and D.-C. Tang, "13th five-year plan textbook of Traditional Chinese Medicine Pharmacy," China Press of Traditional Chinese Medicine, vol. 2, no. 2, pp. 53-59, 2016.

[42] C. Peng, Chinese Dictionary of Clinical Drugs, China Medical Science Press, Beijing, China, 2018.

[43] O. Trott and A. J. Olson, "AutoDock Vina: improving the speed and accuracy of docking with a new scoring function, efficient optimization, and multithreading," Journal of Computational Chemistry, vol. 31, no. 2, pp. 455-461, 2010.

[44] L. J. Le, I. W. Chao, H. Q. Yong, X. B. Wen, and Q. Yi, "Molecular mechanism of diyu shengbai tablets in treatment of leukopenia based on network pharmacology," Modern Chinese Medicine, vol. 22, no. 9, pp. 1-16, 2007.

[45] M. A. Iñiguez, A. Rodŕiguez, O. V. Volpert, M. Fresno, and J. M. Redondo, "Cyclooxygenase-2: a therapeutic target in angiogenesis," Trends in Molecular Medicine, vol. 9, no. 2, pp. 73-78, 2005.

[46] R. S. Apte, D. S. Chen, and N. Ferrara, "VEGF in signaling and disease: beyond discovery and development," Cell, vol. 176, no. 6, pp. 1248-1264, 2019.

[47] J. E. Rundhaug, "Matrix metalloproteinases and angiogenesis," Journal of Cellular and Molecular Medicine, vol. 9, no. 2, pp. 267-285, 2005.

[48] M. Hollborn, C. Stathopoulos, A. Steffen, P. Wiedemann, L. Kohen, and A. Bringmann, "Positive feedback regulation between MMP-9 and VEGF in human RPE cells," Investigative Opthalmology \& Visual Science, vol. 48, no. 9, p. 4360, 2007.

[49] I. Stamenkovic, "Extracellular matrix remodelling: the role of matrix metalloproteinases," The journal of Pathology: a Journal of the Pathological Society of Great Britain and Ireland, vol. 200, no. 4, pp. 448-464, 2003.

[50] A. Yabluchanskiy, Y. Ma, R. P. Iyer, M. E. Hall, and M. L. Lindsey, "Matrix metalloproteinase-9: many shades of function in cardiovascular disease," Physiology, vol. 28, no. 6, pp. 391-403, 2013.

[51] H. Huang, "Matrix metalloproteinase-9 (MMP-9) as a cancer biomarker and MMP-9 biosensors: recent advances," Sensors, vol. 18, no. 10, p. 3249, 2021.

[52] Z. Ma, T. Piao, Y. Wang, and J. Liu, “Astragalin inhibits IL$1 \beta$-induced inflammatory mediators production in human osteoarthritis chondrocyte by inhibiting NF- $\kappa \mathrm{B}$ and MAPK activation," International Immunopharmacology, vol. 25, no. 1, pp. 83-87, 2015.

[53] N. Azoitei, A. Becher, K. Steinestel et al., "PKM2 promotes tumor angiogenesis by regulating HIF- $1 \alpha$ through NF- $\kappa \mathrm{B}$ activation," Molecular Cancer, vol. 15, no. 1, 2016.

[54] M. Imran, B. Salehi, J. Sharifi-Rad et al., "Kaempferol: a key emphasis to its anticancer potential," Molecules, vol. 24, no. 12, 2277 pages, 2017.

[55] Y.-W. Kim and T. V. Byzova, "Oxidative stress in angiogenesis and vascular disease," Blood, vol. 123, no. 5, pp. 625-631, 2014.

[56] W. N. Duran, J. W. Breslin, and F. A. Sanchez, "The NO cascade, eNOS location, and microvascular permeability," Cardiovascular Research, vol. 87, no. 2, pp. 254-261, 2010.

[57] J. Lu, L. V. Papp, J. Fang, S. Rodriguez-Nieto, B. Zhivotovsky, and A. Holmgren, "Inhibition of mammalian thioredoxin reductase by some flavonoids: implications for myricetin and quercetin anticancer activity," Cancer Research, vol. 66, no. 8, pp. 4410-4418, 2006.

[58] J. Lu and A. Holmgren, "The thioredoxin antioxidant system," Free Radical Biology and Medicine, vol. 66, pp. 75-87, 2014.

[59] D. Lee, I. M. J. Xu, D. K. C. Chiu et al., "Induction of oxidative stress through inhibition of thioredoxin reductase 1 is an effective therapeutic approach for hepatocellular carcinoma," Hepatology, vol. 69, no. 4, pp. 1768-1786, 2019.

[60] J. Zhang, J. Yao, S. Peng, X. Li, and J. Fang, "Securinine disturbs redox homeostasis and elicits oxidative stress-mediated apoptosis via targeting thioredoxin reductase," Biochimica et Biophysica Acta (BBA) Molecular Basis of Disease, vol. 1863, no. 1, pp. 129-138, 2017. 
[61] M. A. Abdelsaid, S. Matragoon, and A. B. El-Remessy, "Thioredoxin-Interacting protein expression is required for VEGF-mediated angiogenic signal in endothelial cells," $A n-$ tioxidants \& Redox Signaling, vol. 19, no. 18, pp. 2199-2212, 2013.

[62] L. L. Dunn, A. M. Buckle, J. P. Cooke, and M. K. C. Ng, "The emerging role of the thioredoxin system in angiogenesis," Arteriosclerosis, Thrombosis, and Vascular Biology, vol. 30, no. 11, pp. 2089-2098, 2010.

[63] Q. Yang, D. Yu, and Y. Zhang, “ $\beta$-Sitosterol attenuates the intracranial aneurysm growth by suppressing TNF$\alpha$-mediated mechanism," Pharmacology, vol. 104, no. 6, pp. 302-310.

[64] W.-H. Hu, G. K.-L. Chan, J.-S. Lou et al., "The extract of Polygoni Cuspidati Rhizoma et Radix suppresses the vascular endothelial growth factor-induced angiogenesis," Phytomedicine, vol. 42, pp. 135-143, 2019.

[65] P. Abeyrathna and Y. Su, "The critical role of Akt in cardiovascular function," Vascular Pharmacology, vol. 74, pp. 38-48, 2019.

[66] Q. Zhang, S. Lu, T. Li et al., "ACE2 inhibits breast cancer angiogenesis via suppressing the VEGFa/VEGFR2/ERK pathway," Journal of Experimental \& Clinical Cancer Research, vol. 38, no. 1, 2019.

[67] N. Panth, K. R. Paudel, D.-S. Gong, and M.-H. Oak, "Vascular protection by ethanol extract of Morus alba Root Bark: endothelium-dependent relaxation of rat aorta and decrease of smooth muscle cell migration and proliferation," EvidenceBased Complementary and Alternative Medicine, vol. 2018, Article ID 7905763, 8 pages, 2018.

[68] S. C. Bir, Y. Xiong, C. G. Kevil, and J. Luo, "Emerging role of PKA/eNOS pathway in therapeutic angiogenesis for ischaemic tissue diseases," Cardiovascular Research, vol. 95, no. 1, pp. 7-18, 2012.

[69] J. R. Molina and A. A. Adjei, "The ras/raf/MAPK pathway," Journal of Thoracic Oncology, vol. 1, no. 1, pp. 7-9, 2006.

[70] J. Bao, R. Ding, L. Zou et al., "Forsythiae fructus inhibits B16 melanoma growth involving MAPKs/Nrf2/HO-1 mediated anti-oxidation and anti-inflammation," The American Journal of Chinese Medicine, vol. 44, no. 5, pp. 1043-1061, 2016.

[71] J. Nugent-Head, "Returning our focus to the flavour and nature of herbs," The Journal of Chinese Medicine, vol. 105, p. 30, 1870.

[72] P. Shi, Y. Cao, J. Gao et al., "Allicin improves the function of cardiac microvascular endothelial cells by increasing PECAM-1 in rats with cardiac hypertrophy," Phytomedicine, vol. 51, pp. 241-254, 2018.

[73] Y. Zang, J. Wan, Z. Zhang, S. Huang, X. Liu, and W. Zhang, "An updated role of astragaloside IV in heart failure," Biomedicine \& Pharmacotherapy, vol. 126, Article ID 110012, 2020.

[74] Q. Wang, C.-P. Su, H.-M. Zhang, Y.-L. Ren, W. Wang, and S.-Z. Guo, "Anti-inflammatory mechanism of heat-clearing and detoxifying Chinese herbs," China journal of Chinese Materia Medica, vol. 43, no. 18, pp. 3787-3794, 2018.

[75] T. Liu, X. Liu, and W. Li, "Tetrandrine, a Chinese plantderived alkaloid, is a potential candidate for cancer chemotherapy," Oncotarget, vol. 7, no. 26, Article ID 40800, 2016.

[76] R. Mancuso, M. Foglio, and S. O. Saad, "Artemisinin-type drugs for the treatment of hematological malignancies," Cancer Chemotherapy and Pharmacology, vol. 87, no. 1, pp. 1-22, 2021.

[77] J. C. W. Lim, T. K. Chan, D. S. Ng, S. R. Sagineedu, J. Stanslas, and W. F. Wong, "Andrographolide and its analogues: versatile bioactive molecules for combating inflammation and cancer," Clinical and Experimental Pharmacology and Physiology, vol. 39, no. 3, pp. 300-310, 2012.

[78] C. Wu, J. Chen, C. Chen et al., "Wnt/ $\beta$-catenin coupled with HIF-1 $\alpha$ /VEGF signaling pathways involved in galangin neurovascular unit protection from focal cerebral ischemia," Scientific Reports, vol. 5, no. 1, pp. 1-11, 2015.

[79] X. Ai, P. Yu, Y. Hou et al., "A review of traditional Chinese medicine on treatment of diabetic retinopathy and involved mechanisms," Biomedicine \& Pharmacotherapy, vol. 132, Article ID 110852, 2020.

[80] T.-F. Tzeng, W. Y. Liu, S.-S. Liou, T.-Y. Hong, and I.-M. Liu, "Antioxidant-rich extract from plantaginis semen ameliorates diabetic retinal injury in a streptozotocin-induced diabetic rat model," Nutrients, vol. 8, no. 9, p. 572, 2011. 